\title{
Urinary Retention and Severe Hyponatremia: An Unusual Presentation of COVID-19
}

\author{
Ahmed Osman Saleh ${ }^{1}$ Shaikha D Al-Shokri ${ }^{1}$, Ashraf OE Ahmed ${ }^{1}$, Ahmed Elmustafa Musa ${ }^{2}$, Mouhand FH Mohamed ${ }^{1}$ \\ ${ }^{1}$ Internal Medicine Department, Medical Education, Hamad Medical Corporation, Doha, Qatar \\ ${ }^{2}$ Diagnostic and Interventional Radiology Department, Medical Education, Hamad Medical Corporation, Doha, Qatar
}

\section{Doi: 10.12890/2020_001905 - European Journal of Case Reports in Internal Medicine - ๑ EFIM 2020}

Received: $22 / 07 / 2020$

Accepted: $14 / 08 / 2020$

Published: $11 / 09 / 2020$

How to cite this article: Saleh AO, Al-Shokri SD, Ahmed AOE, Musa AE, Mohamed MFH. Urinary retention and severe hyponatremia: an unusual presentation of COVID-19. EJCRIM 2020;7: doi:10.12890/2020_001905.

Conflicts of Interests: The Authors declare that there are no competing interests.

Acknowledgements: We thank Qatar National Library for funding the open access publication fees of this article. Additionally, we thank the critical care, medical, neurology and infectious disease personnel involved in the care of this patient.

This article is licensed under a Commons Attribution Non-Commercial 4.0 License

\section{ABSTRACT}

Coronavirus disease 2019 (COVID-19) was first reported in Wuhan, China, in December 2019. The disease is caused by severe acute respiratory syndrome virus coronavirus 2 (SARS-CoV-2). A few published cases have linked COVID-19 and hyponatremia. The mechanism of hyponatremia in these cases is related to the syndrome of inappropriate antidiuretic hormone secretion (SIADH). Here we present a unique case of urinary retention and SIADH as unusual presenting features of SARS-CoV-2 infection.

\section{LEARNING POINTS}

- $\quad$ Urine retention could be an unusual presenting symptom of severe hyponatremia of COVID-19.

- Hyponatremia and SIADH could be unusual presenting features of SARS-CoV-2 infection.

- Careful correction of hyponatremia related to COVID-19 is necessary to avoid osmotic demyelination syndrome.

\section{KEYWORDS}

COVID-19, coronavirus, SARS-CoV-2, hyponatremia, SIADH, urine retention

\section{INTRODUCTION}

Coronavirus disease 2019 (COVID-19) started in Wuhan, China, and spread globally. It is caused by severe acute respiratory syndrome virus coronavirus 2 (SARS-CoV-2) ${ }^{[1]}$. The clinical presentation is variable with a predominance of mild respiratory symptoms ${ }^{[2,3]}$. Syndrome of inappropriate antidiuretic hormone secretion (SIADH) has been associated with different pulmonary infections and was described in a few cases related to COVID-19, but there are no reports linking urinary retention to COVID-19 or hyponatremia in the literature ${ }^{[4,5]}$. Neurological manifestations including stroke, a drop in the level of consciousness, loss of smell, ataxia and convulsions have been associated with COVID-19, but there have been no reports linking urinary retention to COVID-19 ${ }^{[6,7]}$. We describe a patient with COVID-19 who presented unusually with urinary retention and SIADH.

\section{CASE DESCRIPTION}

We present the case of a 52-year-old man known to have hypertension and on oral amlodipine. He presented with a 10-day history of progressive suprapubic pain, dysuria, urinary hesitancy, inability to pass urine, and generalized weakness. Further inquiry revealed a 3-week history of loose non-bloody stools occurring three times per day and associated with nausea and vomiting. He did not have a fever, flu 
symptoms, cough, shortness of breath, change in consciousness, focal weakness, abnormal movements, or seizures. There was no history of recent travel or contact with sick individuals.

Upon initial examination, the patient was confused and responding slowly to commands. He was not febrile, tachypnoeic or tachycardic. Assessment of volume status revealed no evidence of dehydration or hypotension. Abdominal examination showed suprapubic fullness and tenderness. There were no focal neurological deficits and the chest and cardiovascular examinations were unremarkable. Bedside ultrasound (US) showed urinary retention estimated at 1.2 litres with no hydronephrosis. Formal US assessment showed that the prostate was the normal size and shape, and measured $23 \mathrm{ml}$ in volume.

Initial laboratory investigations were remarkable for severe hyponatremia of $103 \mathrm{mmol} / \mathrm{l}$ (135-145 mmol/I) consistent with SIADH (Table 1).

\begin{tabular}{|c|c|c|}
\hline Test & Value & Normal Range \\
\hline Ferritin & $1,053.0 \mu g / l$ & $30.0-553.0$ \\
\hline Triglyceride & $1.0 \mathrm{mmol} / \mathrm{l}$ & $<1.7$ \\
\hline Urine osmolality & $391 \mathrm{mmol} / \mathrm{kg}$ & $<100$ \\
\hline Urine sodium & $68 \mathrm{mmol} / \mathrm{l}$ & $<40$ \\
\hline WBC & $8.9 \times 103 / \mu l$ & $4.0-10.0$ \\
\hline Haemoglobin & $17.0 \mathrm{~g} / \mathrm{dl}$ & $13.0-17.0$ \\
\hline Lymphocyte Auto-numbering & $0.5 \times 103 / \mu l$ & $1.0-3.0$ \\
\hline Urea & $3.4 \mathrm{mmol} / \mathrm{l}$ & $2.8-8.1$ \\
\hline Creatinine & $90 \mu \mathrm{mol} / \mathrm{l}$ & $62-106$ \\
\hline Potassium & $2.9 \mathrm{mmol} / \mathrm{l}$ & $3.5-5.1$ \\
\hline Chloride & $67 \mathrm{mmol} / \mathrm{l}$ & $98-107$ \\
\hline Bicarbonate & $25 \mathrm{mmol} / \mathrm{l}$ & $22-29$ \\
\hline Calcium & $2.19 \mathrm{mmol} / \mathrm{l}$ & $2.15-2.50$ \\
\hline Calcium (corrected) & $2.31 \mathrm{mmol} / \mathrm{l}$ & $2.15-2.50$ \\
\hline Total protein & $70 \mathrm{~g} / \mathrm{l}$ & $66-87$ \\
\hline Albumin level & $34 \mathrm{~g} / \mathrm{l}$ & $35-52$ \\
\hline Alkaline phosphatase & $84 \mathrm{U} / \mathrm{I}$ & $40-129$ \\
\hline ALT & $81 \mathrm{U} / \mathrm{I}$ & $0-41$ \\
\hline AST & $43 \mathrm{U} / \mathrm{I}$ & $0-40$ \\
\hline Osmolality & $225 \mathrm{mmol} / \mathrm{kg}$ & $275-295$ \\
\hline Glucose & $7.5 \mathrm{mmol} / \mathrm{l}$ & $3.3-5.5$ \\
\hline CRP & $52.8 \mathrm{mg} / \mathrm{l}$ & $0.0-5.0$ \\
\hline Cortisol & $557.0 \mathrm{nmol} / \mathrm{I}$ & $68.2-327$ \\
\hline TSH & $0.92 \mathrm{mIU} / \mathrm{l}$ & $0.30-4.20$ \\
\hline FT4 & $20.0 \mathrm{pmol} / \mathrm{l}$ & $11.6-21.9$ \\
\hline Venous sodium (point-of-care) & $103 \mathrm{mmol} / \mathrm{l}$ & $135-145$ \\
\hline
\end{tabular}

Table 1. Laboratory results

The initial chest $\mathrm{x}$-ray was unremarkable. Because of his neurological symptoms, the patient was given a bolus of $3 \%$ saline $50 \mathrm{ml}$ and then was started on $50 \mathrm{ml} / \mathrm{h}$ (receiving a total of $150 \mathrm{ml}$ ) in the emergency department. The sodium level was monitored periodically and increased from 103 to $114 \mathrm{mmol} / \mathrm{l}$, and so hypertonic saline was stopped to avoid further increase and rapid correction. The patient had high inflammatory markers, lymphopenia, thrombocytopenia, and a positive nasopharyngeal SARS-CoV-2 RT-PCR test. He was started on COVID-19 treatment as per protocol, and Foley's catheter was inserted to relieve urinary retention. 
Despite sodium correction, the patient remained confused and disoriented. His Glasgow Coma Scale (GCS) dropped from 14 to 8 and so he was moved to the critical care unit. An extensive investigation was done to evaluate the drop in his level of consciousness; a CT scan of the head and MRI were normal, and CSF analysis, and CSF and blood cultures were negative for infections. An MRI of the brain repeated 10 days later showed features of osmotic demyelination syndrome (Fig. 1). The patient remained in the critical care unit under supportive care, on a feeding nasogastric tube and Foley's catheter. After clinical stabilization he was moved to a rehabilitation institute and improved significantly. Currently he has no urinary symptoms, can eat a soft diet and can ambulate independently, but requires assistance with climbing stairs. Upon further questioning, he reported loss of taste and smell for 1 week but denied urinary symptoms prior to his illness. His international prostate symptom score (I-PSS) was zero, which means he was completely asymptomatic.

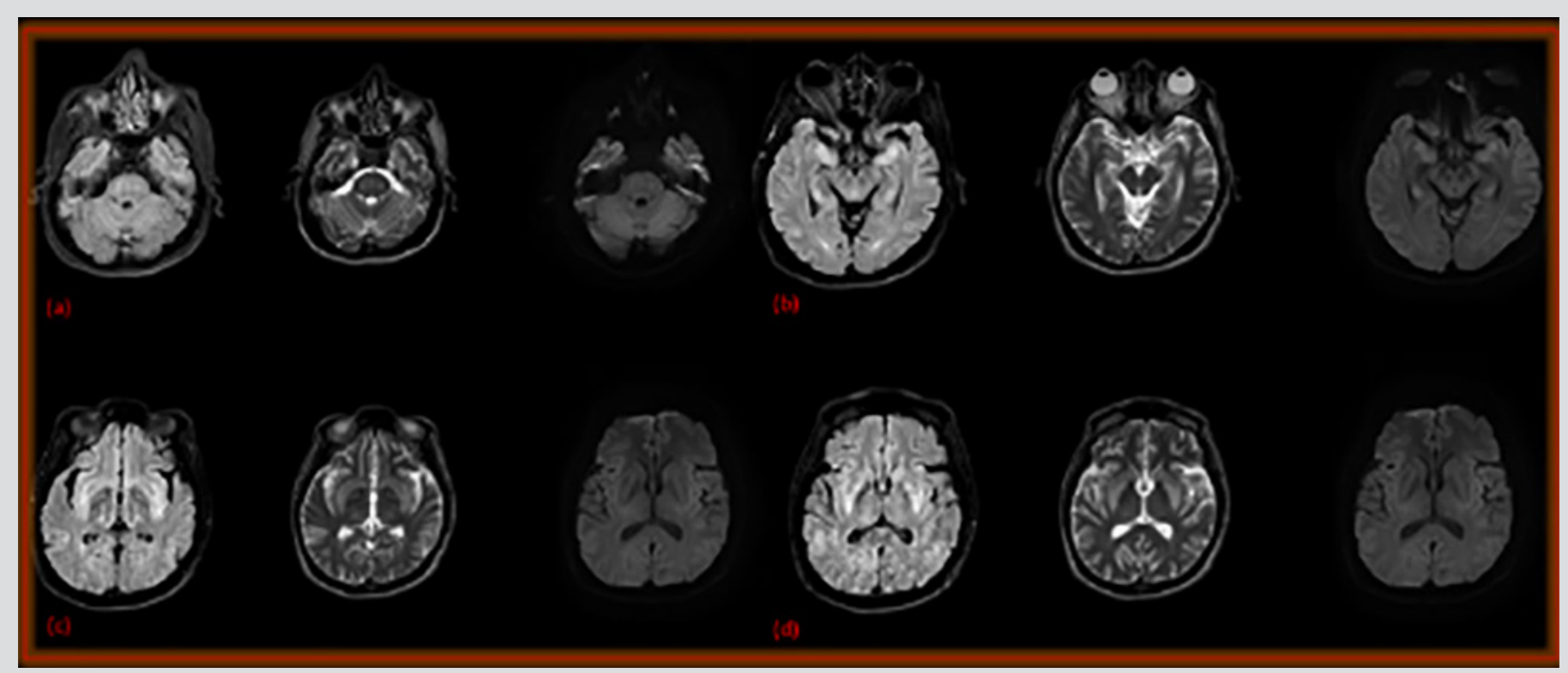

Figure 1. Axial FLAIR and DWI at the level of the pons (a) hippocampus (b), insula (c) and basal ganglia (d), showing bilateral, symmetrical, abnormal, bright FLAIR/T2 signal intensity

\section{DISCUSSION}

The novel coronavirus is one of the severe acute respiratory syndrome-related coronaviruses. The most common clinical characteristics are fever, dry cough, myalgia, anorexia and dyspnoea. Gastrointestinal symptoms such as abdominal pain, vomiting and diarrhoea have been reported as less common manifestations of COVID-19 ${ }^{[3]}$. Recent data from the USA showed that hyponatremia occurred in $50 \%$ of patients admitted with COVID-19 ${ }^{[7]}$. Another cause of hyponatremia in patients with COVID-19 is the increased release of antidiuretic hormone $(A D H)$, which retains water and dilutes the sodium in the blood. Alternatively, SIADH can occur in response to numerous comorbidities caused by COVID-19, including pneumonia and respiratory failure. In our patient, vomiting and diarrhoea could explain the hyponatremia and hypokalaemia. However, the low serum osmolarity coupled with inappropriate natriuresis in the setting of severe hyponatremia was suggestive of SIADH. We ruled out other potential causes of SIADH, and so SIADH, in this case, was likely due to COVID-19 (Table 1).

Urinary retention as a cause of hyponatremia was reported in elderly patients with a mean age of 85 years. In these patients, the mean serum sodium level was $120 \mathrm{mEq} / \mathrm{I}$ and completely normalized after urinary catheterization, and thus was explained by urinary outlet obstruction ${ }^{[8]}$. Our patient presented with urinary retention for the first time, but his normal US and I-PSS score of zero excluded urinary outlet obstruction as a possible cause of his hyponatremia and urinary retention. Additionally, urinary retention in critically ill patients can be explained by bed confinement and the use of hypnotics in hospital ${ }^{[9]}$. This was not the case in our patient who denied difficulty ambulation at home, and urinary retention was discovered in the emergency department.

Neurological manifestations including stroke, a drop in the level of consciousness, loss of smell, ataxia and convulsions have been linked to COVID-19 ${ }^{[6,7]}$. After he improved, our patient reported that he had loss of taste and smell few days prior to his presentation to hospital, but he denied weakness, difficulty ambulation or other neurological symptoms. This case highlighted the fact that urinary retention could be an unusual presentation of SARS-CoV-2 infection. This may be due to the direct effect of SARS-CoV-2. This is supported by previous studies that reported neuroinvasive properties of SARS-CoV-2. Haematogenous spread and entry via neuronal retrograde dissemination were the two possibilities, the latter of which could also explain anosmia ${ }^{[6,10]}$. 
The presence of anosmia, dysgeusia and a drop in the level of consciousness despite sodium correction may indicate that urinary retention in our patient was likely a neurological manifestation of COVID-19. CSF SARS-CoV-2 PCR testing could be a useful investigation to support the possibility of neuroinvasion, but this was not done in our patient. On the other hand, severe hyponatremia is associated with neurological symptoms, such as weakness, change in consciousness level, and depressed reflexes ${ }^{[11]}$. Inhibition of the urinary reflex arc secondary to severe hyponatremia is another possible cause of urinary retention that is not well explored in the literature. Nonetheless, as more data become available, we will understand this association better and its management implications.

Patients with severe hyponatremia are at risk of cerebral oedema if the hyponatremia is under-corrected, and may develop osmotic demyelination from rapid or over-correction. Our patient was treated with hypertonic saline (3\%), but despite the correction of hyponatremia, his neurological symptoms did not improve. He received $150 \mathrm{ml}$ of $3 \%$ saline only and had a normal initial MRI of the brain, so osmotic demyelination was deemed less likely, and thus other potential causes were investigated and excluded. Despite the significant sodium deficit and the fact that the patient received only $150 \mathrm{ml}$ of $3 \%$ saline, osmotic demyelination was evident on repeat MRI brain 10 days later (Fig. 1). This could indicate that hyponatremia associated with COVID-19 may respond differently to treatment; hence more frequent sodium level monitoring might be necessary.

\section{CONCLUSION}

During the ongoing COVID-19 pandemic, hyponatremia and SIADH could be unusual presenting features of SARS-CoV-2 infection. Raising awareness about this association may lead to faster diagnosis and management. Urine retention could also be a presenting symptom of COVID-19 or severe hyponatremia; however, more reports are needed to explore this association further. To avoid osmotic demyelination syndrome, we suggest more frequent sodium level monitoring in patients with COVID-19-related-SIADH treated with hypertonic saline.

\section{REFERENCES}

1. World Health Organization. Novel Coronavirus (2019-nCoV) Situation Report - 1. 21 January 2020.

2. Centers for Disease Control and Prevention. Symptoms of coronavirus. https://www.cdc.gov/coronavirus/2019-ncov/symptoms-testing/symptoms.html (accessed 19 August 2020).

3. Wang D, Hu B, Hu C, Zhu F, Liu X, Zhang J, et al. Clinical characteristics of 138 hospitalized patients with 2019 novel coronavirus-infected pneumonia in Wuhan, China. JAMA 2020;323:1061-1069.

4. Yousaf Z, Al-Shokri SD, Al-Soub H, Mohamed MFH. COVID-19-associated SIADH: a clue in the times of pandemic! Am J Physiol Endocrinol Metab 2020;318(6):E882-E885.

5. Habib MB, Sardar S, Sajid J. Acute symptomatic hyponatremia in setting of SIADH as an isolated presentation of COVID-19. IDCases 2020;21:e00859.

6. Baig AM. Neurological manifestations in COVID-19 caused by SARS-CoV-2. CNS Neurosci Ther 2020;26(5):499-501.

7. Aggarwal S, Garcia-Telles N, Aggarwal G, Lavie C, Lippi G, Henry B. Clinical features, laboratory characteristics, and outcomes of patients hospitalized with coronavirus disease 2019 (COVID-19): early report from the United States. Diagnosis 2020;7(2):91-96.

8. Galperin I, Friedmann R, Feldman H, Sonnenblick M. Urinary retention: a cause of hyponatremia? Gerontology 2007;53(3):121-124.

9. Schettini DA, Freitas FG, Tomotani DY, Alves JC, Bafi AT, Machado FR. Incidence and risk factors for urinary retention in critically ill patients. Nurs Crit Care 2018;24(6):355361.

10. Shang J, Wan Y, Luo C, Ye G, Geng Q, Auerbach A, et al. Cell entry mechanisms of SARS-CoV-2. Proc Natl Acad Sci USA 2020;117(21):11727-11734.

11. Giuliani C, Peri A. Effects of hyponatremia on the brain. J Clin Med 2014;3(4):1163-1177. 